\title{
Evaluation of vibration on technological devices
}

Petr Lukovics

Brno University of Technology, Faculty of Mechanical Engineering, Czech Republic. E-mail: petrlukovics@seznam.cz

Keywords: Vibration, Grinding, Surface quality, Roughness

Research paper discuss the possibility of vibration detection on production machine and also presents devices and methodology for evaluation of vibration amplitude using non-contact laser interferometer and contact piezoelectric vibration sensors. Experimentally determines the influence of technological conditions on the quality of functional surfaces. Furthermore, paper evaluates the influence of technological conditions during planar grinding on the vibration amplitude of the grinding spindle and presents correlation between grinder vibration amplitude and quality of the product.

\section{References}

[1] BÍLEK, O., LUKOVICS, I. Model of Dynamics within Highspeed Grinding Process. In DUSE, D.M. ; BRINDASU, P.D.; BEJU, L.D. (eds.). MSE 2009: Proceedings of the Manufacturing Science and Education. Sibiu, Romania, June 4-6. Sibiu: Lucian Blaga University of Sibiu, 2009, p. 11-14. ISSN 1843-2522.

[2] HOLEŠOVSKÝ, F., NOVÁK, M., MICHNA, Š. Studium změn broušené povrchové vrstvy při dynamickém zatěžování. Strojírenská technologie. s. 73-76. ISSN 1211-4162.

[3] JURKO, J., LUKOVICS, I. Obrábatel’nost’ materiálov. 1.vyd, Zlín: UTB, 2008. 144p. ISBN 978-80-7318-7361.

[4] KUNDRÁK, J. Alternative machining procedures of hardened steels. Manufacturing technology. 2011, vol 11, no. 11., pp. 32-39, ISSN 1213-2489.

[5] LUKOVICS, I., BÍLEK, O. High Speed Grinding Process. Manufacturing Technology, 2008, 8, 12-18. ISSN 1213248-9.

[6] LUKOVICS, I., BÍLEK, O. Precise Grinding. 7th International Tools Conference. Zlín, 3.-4.2.2009. ISBN 97880-7318-794-1.

[7] MONKA, P., MONKOVA, K., ZAJAC, J. Multi Variant Process Plans. In. DAAAM International Scientific Book 2009, Vienna : DAAAM Int., 2009, ISBN 978-3-901509-69-8.

[8] NOVÁK, M. Možnosti hodnocení kvality obrobených povrchů. Strojírenská technologie. 2010, zvl. vydání, Ústí n. Labem : FVTM UJEP, s. 195-198. ISSN 1211-4162.

[9] NOVÁK, P., MÁDL, J. Effective Evaluation of Measured Dynamic Values of Cutting Forces and Torques. Manufacturing Technology, vol. I, 2001, pp 56-62, ISSN 1213248-9.

[10] PANDA, A., JURKO, J., KURILOVSKÝ, T. Výroba pre automobilový priemysel. In 15th International scientific conference: Quality and reliability of technical systems. 2010. pp. 377-382. ISBN 978-80-552-0390-4.7

[11] KUNDRAK, J., MAMALIS, A. G., GYANI, K., BANA, V. Surface layer microhardness changes with highspeed, The International Journal of Advanced Manufacturing Technology. Volume 53, Issue 1-4 (2011), pp.105-112 DOI: 10.1007/s00170-010-2840-y

[12] LUKOVICS, I., ROKYTA, L. Influence of the Technological Conditions on Quality by Grinding. Strojirenská technologie, 2010, roč. 14, s. 151-154. ISSN 1211-4162

[13] LUKOVICS, I., BÍLEK, O., HOLEMÝ, S., Development of Grinding Wheels for Tools Manufacturing. Manufacturing Technology, 2010, č.10, s.10-16. ISSN 1213-2489 